Research Paper

\title{
Predictive Value of Primary Tumor Site for Loco- regional Recurrence in Early Breast Cancer Patients with One to Three Positive Axillary Lymphadenophy
}

\author{
Shaoqing Niu1,2,3*, Ge Wen ${ }^{1,2,4^{*}}$, Yufeng Ren $3^{*}$, Yiyang Li1,2,5, Lingling Feng1,2, Chengtao Wang3, Xiaobo \\ Huang $^{1,2}$, Bixiu Wen ${ }^{3 凶}$, Yujing Zhang ${ }^{1,2} \bowtie$ \\ 1. Sun Yat-sen University Cancer Center; State Key Laboratory of Oncology in South China; Collaborative Innovation Center for Cancer Medicine, \\ Guangzhou, Guangdong 510060, P. R. China \\ 2. Department of Radiation Oncology, Sun Yat-sen University Cancer Center, Guangzhou, Guangdong 510060, P. R. China \\ 3. Department of Radiation Oncology, the First Affiliated Hospital of Sun Yat-sen University, Guangzhou, Guangdong 510060, P.R. China \\ 4. Department of Radiation Oncology, the Third Affiliated Hospital of Guangzhou Medical University, Guangzhou, Guangdong 510150, P. R. China \\ 5. Department of Oncology, the First Affiliated Hospital of Guangdong Pharmaceutical University, Guangzhou, Guangdong 510060, P.R. China \\ *The three authors contributed equally to this article.
}

$\square$ Corresponding authors: Yujing Zhang, M.D., Ph.D. Department of Radiation Oncology, State Key Laboratory of Oncology in South China, Sun Yat-sen University Cancer Center, Guangzhou 510060, China. E-mail: zhangyj@sysucc.org.cn; Tel: (8620) 87343702; Fax: (8620) 87343392 and Bixiu Wen, M.D., Ph.D. Department of Radiation Oncology, the First Affiliated Hospital of Sun Yat-sen University, Guangzhou 510080, China. E-mail: wenbix@mail.sysu.edu.cn; Tel: (8620) 87755766 x8425; Fax:(8620)87750632

(c) Ivyspring International Publisher. This is an open access article distributed under the terms of the Creative Commons Attribution (CC BY-NC) license (https://creativecommons.org/licenses/by-nc/4.0/). See http://ivyspring.com/terms for full terms and conditions.

Received: 2017.02.18; Accepted: 2017.05.13; Published: 2017.07.22

\begin{abstract}
Introduction: It remains controversial on high risks for early breast cancer patients with one to three axillary nodes after mastectomy who is predisposition to locoregional recurrence. The present study is to investigate the relationship between primary tumor site and loco-regional recurrence (LRR) and explore the predictive value of clinicopathological characteristics in LRR for early breast cancer patients with one to three positive axillary lymph nodes after mastectomy.

Methods: We reviewed the clinical data of 656 consecutively diagnosed patients with $\mathrm{pT}_{1-2} \mathrm{~N}_{1} \mathrm{M}_{0}$ breast cancer who were treated in Sun Yat-sen University Cancer Center with radical operation without postoperative radiotherapy between March 1998 and December 2010. The primary tumor sites included outer quadrant in 455 patients (69.36\%), inner quadrant in 156 patients (23.78\%)and central quadrant in 45 patients (6.86\%). LRR and LRR-free survival (LRFS) in combination with clinical and pathological features were analyzed to screen out patients with higher risk of LRR.

Results: The median follow-up time was 64.9 months. The 5-, 10-year LRR for the cohort was $8.6 \%$ and $12.9 \%$, respectively; the 5 -, 10-year LRFS was $86.2 \%$ and $76.4 \%$, respectively. Multivariate analyses showed that age of $\leq 35$ years, inner quadrant tumor and non-luminal subtype were independent risk factors for LRR and LRFS. Patients with primary tumor in inner quadrant showed higher LRR and poorer LRFS when risk factors are $\geq 2$ than those with tumors in other sites.

Conclusions: Inner quadrant tumor was an independent predictor for LRR and LRFS in patients with early breast cancer and one to three positive axillary lymph nodes, which would be more accurate in combination with other prognostic indexes including patients' age, pathological T stage, Ki67 status, molecular subtypes.
\end{abstract}

Key words: Early breast cancer; Primary tumor site; Axillary lymph node; Loco-regional recurrence; Risk factors.

\section{Introduction}

Whether patients with $\mathrm{pT}_{1-2} \mathrm{~N}_{1} \mathrm{M}_{0}$ breast cancer treated with radical mastectomy or modified radical mastectomy could benefit from postoperative radiotherapy has not been confirmed by large 
prospective randomized clinical study [1-11]. The Early Breast Cancer Trialists' Collaborative Group (EBCTCG) [12] in 2014 has reported that post-mastectomy radiotherapy to patients with one to three axillary nodes reduced recurrence and breast cancer mortality even when patients were administered with systemic chemotherapy in a meta-analysis. However, it still remains controversial on high risks for early breast cancer patients with one to three axillary nodes after mastectomy who is predisposition to locoregional recurrence. The present study is to investigate the relationship between primary tumor site and locoregional recurrence (LRR) and explore the predictive value of clinicopathological characteristics in LRR for early breast cancer patients with one to three positive axillary lymph nodes after mastectomy.

\section{Materials and Methods}

\section{Patient Selection}

Patients with early stage breast cancer who received radical mastectomy or modified radical mastectomy were consecutively recruited between March 1998 and December 2010. All patients were performed pretreatment tests including mammography, ultrasonography on breast and axilla. The inclusion criteria are as follows: (1) Neither neoadjuvant chemotherapy nor other anti-tumor therapy was administered before operation; (2) Standard radical or modified radical mastectomy was performed; (3) No postoperative radiotherapy was delivered; (4) The postoperative pathologic stage was $\mathrm{pT}_{1 \sim 2} \mathrm{~N}_{1} \mathrm{M}_{0}$ according to UICC/AJCC TNM staging classification (2010); (5) The resection margin was negative with complete immunohistochemistry data; (6) No history of other malignancy.

There were 656 patients who were eligible, aging from 23 to 82 years (median 49). Among them, 302 patients $(46.04 \%)$ were premenopausal, 69 (10.52\%) perimenopause and $285(43.44 \%)$ postmenopausal. The primary tumor site was divided into the outer quadrant (OQ), inner quadrant (IQ) and central quadrant (CQ). OQ was defined as the lateral part of bilateral breast, from $12 \mathrm{o}$ 'clock direction to $6 \mathrm{o}$ 'clock in left breast and from 6 o 'clock direction to 12 in right breast. IQ was defined as the interior part of bilateral breast, from 6 o 'clock direction to $12 \mathrm{o}$ 'clock in left breast and $12 \mathrm{o}$ 'clock direction to 6 in right breast. CQ includes the nipple and areola complex.

The primary tumor sites included OQ in 455 patients $(69.36 \%)$, IQ in 156 patients $(23.78 \%)$ and CQ in 45 patients $(6.86 \%)$. The surgical procedure included standard radical mastectomy (Halsted operation) in 104 patients (15.85\%) and modified radical mastectomy (Auchincloss or Patey operation) in 552 patients $(84.15 \%)$.

The axillary dissection has demonstrated with median lymph nodes of 15 ranging from 2 to 42 and 583 patients $(88.87 \%)$ presented with more than 10 axillary lymph nodes. Postoperative pathological diagnosis was made as infiltrating ductal carcinoma in 647 patients $(98.63 \%)$, invasive lobular carcinoma and medullary carcinoma in 9 patients. Lymphovascular invasion was detected in 20 patients (3.05\%).

\section{Pathological parameters}

\section{(1) Estrogen/Progestogen receptors (ER/PR)}

IHC techniques were used to measure ER and PR status from paraffin-embedded tissue sections. ER/PR positive was defined as $\geq 10 \%$ positive cells by IHC in semi-quantitative detection; while ER/PR markers presented in $\geq 1 \%$ was considered to be positive in quantitative detection. The positive expression of ER/PR was detected in 522 patients $(79.57 \%)$ and negative in 134 patients $(20.43 \%)$.

\section{(2) HER-2 evaluation standard}

IHC determined HER-2 expression in tissue. HER-2 positive defined as IHC $(3+)$ or $(2+)$ with confirmation by fluorescence in situ hybridization (FISH). HER-2-positive results were observed in 168 patients $(25.61 \%)$ and HER-2-negative in 471 patients $(71.79 \%)$.

\section{(3) Ki 67 and p53 evaluation standard:}

The expression levels of Ki67 and p53 were determined by IHC analyses. Tissues were fixed in $10 \%$ formaldehyde for 24 hours, then embedded in paraffin and cut into 5-um sections. All sections were adherent to APES coated slides and dried at $60^{\circ} \mathrm{C}$ for 2 hours [13]. Quantitative detection of Ki67 was performed in 270 patients $(41.16 \%)$ and semiquantitative detection in 371 patients (56.55\%). In semi-quantitative detection, the percentage of positive cells $<10 \%$ was considered to be negative, $10 \%-25 \%$ as weak positive $(1+), 26 \%-50 \%$ as positive $(2+)$ and $>50 \%$ as strong positive $(3+)$. Some of patients who received semi-quantitative detection also received quantitative analysis; other 43 patients with semiquantitative detection without quantitative detection were not involved in the statistical analysis of Ki67 expression. 108 patients $(16.46 \%)$ were shown with Ki67 <14\% and 308 (46.95\%) with Ki67 $\geq 14 \%$. Semi-quantitative detection was performed in p53 testing and the evaluation standard was the same with Ki67. There were 156 patients (23.78\%) with p53 $\geq 2+$ and $301(45.88 \%)$ with p53 $<2+$. 


\section{(4) Molecular subtypes}

Tumor subtypes were classified as Luminal subtype (luminal A and luminal B) and non-Luminal subtype [human epidermal growth factor receptor 2 (HER2) enriched and triple-negative (TN) cancers ${ }^{[14]}$. Luminal subtype was defined as ER $(+)$ and/or PR (+) and non-Luminal subtype was defined as ER (-) and PR (-). There were 522 patients $(79.57 \%)$ with luminal subtype and 134 cases (20.43\%) with non-Luminal subtype.

\section{Postoperative chemotherapy}

614 patients received postoperative chemother486 patients $(79.15 \%)$ completed 6 or more cycles of chemotherapy. The regimens for chemotherapy were CAF (cyclophosphamide and epirubicin/doxorubicin and fluorouracil) in 263 patients (42.83\%), TA (docetaxel/taxanes and epirubicin/doxorubicin) in 236 patients $(38.44 \%)$, CMF regimen (cyclophosphamide, methotrexate and fluorouracil) in 40 paitents (6.51\%), CA (cyclophosphamide and epirubicin/ apy with a median of 6 cycles (range, 1-8 cycles) and

doxorubicin) in 32 patients (5.21\%), CMO regimen (cyclophosphamide, mitomycin and vinblastine) in 30 patients $(4.89 \%)$ and other regimen in 13 patients $(2.12 \%)$.

\section{Hormonal therapy}

450 patients received endocrine therapy after mastectomy, including tamoxifen, toremifene or aromatase inhibitors. At the latest follow up, 188 patients $(41.78 \%)$ were treated with endocrine drug less than 3 years, 200 cases $(44.44 \%$ )with 3 to 5 years and 62 cases $(13.78 \%)$ with more than 5 years.

\section{Statistical analysis}

The median follow-up time was 64.9 months. Loco-regional recurrence (LRR), distant metastasis (DM) and survival status were documented. LRR was defined as recurrence presented at the ipsilateral chest wall or axillary, supraclavicular, subclavian or internal mammary lymph node proven histologically, no matter if there was distant metastasis. Primary end points were LRR and LRR-free survival (LRFS). A survival curve was constructed using the
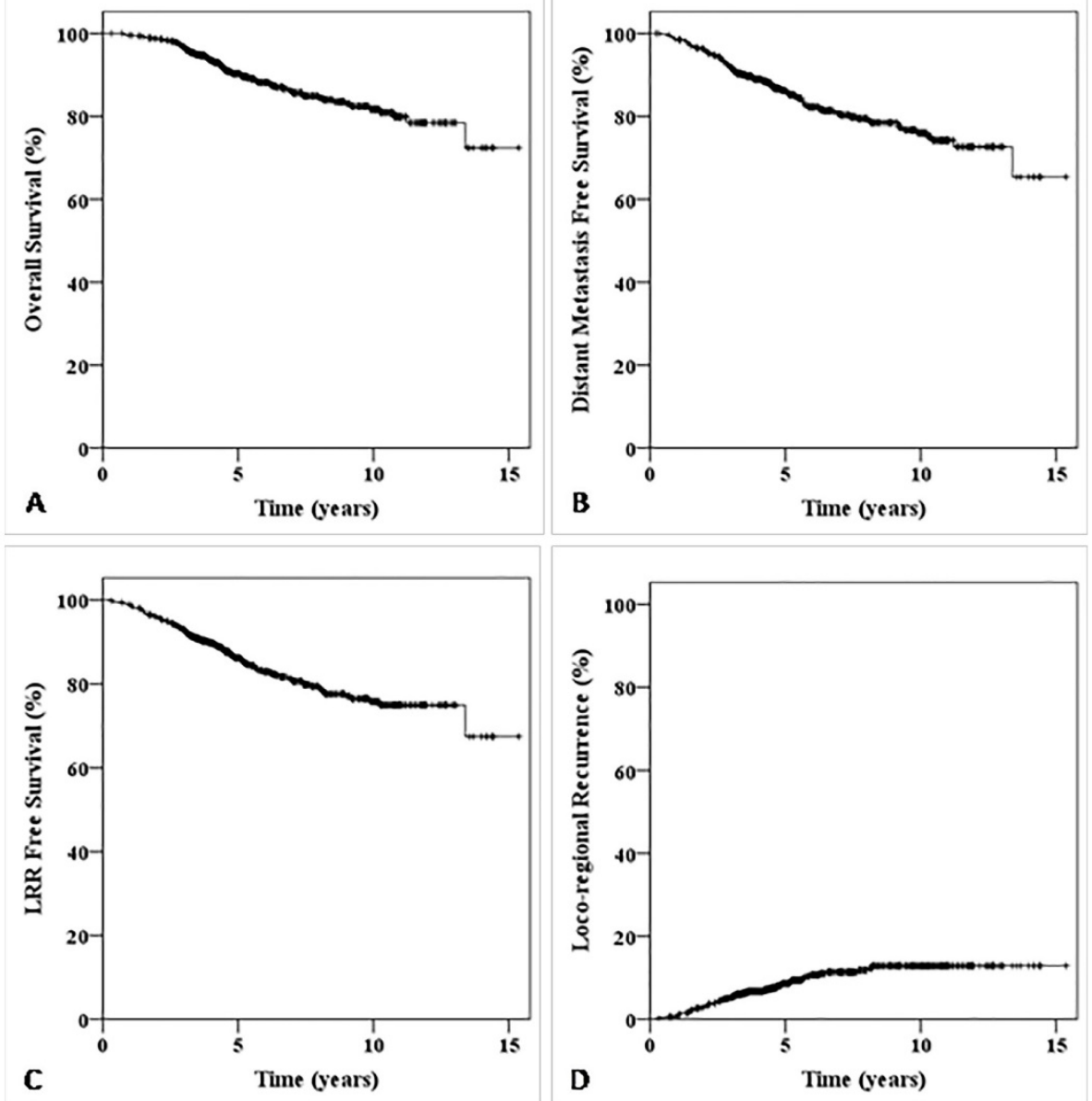

Figure 1. Kaplan-Meier survival curve for all patients in this study. The overall survival (OS) curve for all patients in this study (A); The distant metastasis free survival (DMFS) curve for all patients in this study (B); The LRR free survival (LRFS) curve for all patients in this study (C); The LRR survival curve for all patients in this study (D).
Kaplan-Meier method, and the groups were compared using the log-rank test. $P$ values $<0.05$ were considered as statistically significant. All statistical analyses were performed using SPSS Statistics (Version 19.0).

\section{Results}

\section{The survival and recurrence status}

The overall follow-up rate was $95.3 \%$. There were 416 patients were followed up for more than 5 years and 191 patients for more than 10 years. The 5-, 10-year LRR for all patients is $8.6 \%$ and $12.9 \%$, respectively; the 5-, 10-year LRFS is $86.2 \%$ and $76.4 \%$, respectively (Fig. 1). 82 patients died, including 71 cases $(86.59 \%)$ died of breast cancer, 10 died (12.19\%) of other disease and one died $(1.22 \%)$ of secondary tumor. 116 patients (17.68\%) developed tumor recurrence or distant metastasis. 62 cases $(9.45 \%)$ developed LRR with or without distant metastasis with 
a median LRR time of 32.4 months (rang 2.5 98.4 months), the specific locations of LRR and DM are summarized in Table 1 . The supraclavicular and internal mammary lymphoma nodes (IMLN) LRR rate of patients with primary tumor in inner quadrant was significant higher than that of patients with outer or central quadrant tumor location $(11.4 \%$ vs. $3.7 \%$, $p<0.001)$, but there was no significant difference in chest wall LRR between the two groups.

Table 1. The specific locations of LRR and DM.

\begin{tabular}{lllllllllllll}
\hline $\begin{array}{l}\text { Primary } \\
\text { site }\end{array}$ & No. & \multicolumn{1}{l}{ LRR } & CW & AL & SB & SP & IM & Total* & Bone Lung & Brain Liver & Total \\
\hline IQ & 156 & 8 & 4 & 0 & 18 & 3 & 33 & 10 & 12 & 2 & 10 & 34 \\
CQ & 45 & 1 & 0 & 0 & 1 & 2 & 4 & 1 & 3 & 1 & 2 & 7 \\
OQ & 455 & 13 & 4 & 2 & 16 & 1 & 36 & 28 & 20 & 3 & 16 & 67 \\
\hline
\end{tabular}

Abbreviations: $\mathrm{LRR}=$ Loco-regional recurrence, $\mathrm{DM}=$ distant metastasis,

$\mathrm{IQ}=$ Inner Quadrant, $\mathrm{CQ}=$ Central Quadrant, $\mathrm{OQ}=$ Outer Quadrant; $\mathrm{CW}=$ Chest wall; $\mathrm{AL}=\mathrm{Axillary}$ lymph node; $\mathrm{SB}=$ Subclavian lymph node; $\mathrm{SP}=$ Supraclavicular lymph node; IM=Internal mammary lymph node

* There were 8 patients with two or more sites of LRR and 20 patients with two or more sites of DM.

\section{Prognostic factors}

Clinical and pathological features were evaluated for prognostic significance against LRR and LRFS (Table 2). Univariate analyses indicated that age $\leq 35$, the number of axillary lymph node, stage pT2, Ki67 status, p53 status, non-luminal subtype and inner quadrant tumor were significantly associated with higher risk of LRR $(p<0.05)$. No statistically significant difference was observed in menopausal status (premenopausal/perimenopause vs. postmenopausal, $p=0.344$ ), pathological grade (I/II vs. III, $p=$ 0.351 ), vessel invasion (yes vs. no, $p=0.479$ ) and the number of axillary lymph node detected $(\geq 10 \mathrm{vs} .<10$, $p=0.834$ ).

For LRFS, univariate analyses indicated that age $\leq 35$, pathological stage $\mathrm{pT}_{2}, \mathrm{Ki} 67 \geq 14 \%$ and non-luminal subtype were significantly associated with lower of LRFS $(p<0.05)$. No statistically significant difference was observed in primary tumor site (outer/central quadrant $v s$. inner quadrant, $p=0.073$ ), menopausal status (premenopausal/perimenopause vs. postmenopausal, $p=0.175)$, pathological grade (I/II vs. III, $p=0.188$ ), vessel invasion (yes vs. no, $p=0.169$ ), the number of axillary lymph node ( 1 vs. $2 \sim 3, p=0.214)$ and p53 status $(<2+v s . \geq 2+, p=0.271)$ (Table 2$)$.

Multivariate analysis indicated that age $(\mathrm{HR}=4.510, p=0.042)$, inner quadrant tumor $(\mathrm{HR}=$ 4.014, $p=0.003)$, non-luminal subtype $(\mathrm{HR}=2.749, p=$ $0.046)$ and the number of axillary lymph node $(\mathrm{HR}=3.039, p=0.026)$ were independent risk factors for LRR. Age $(\mathrm{HR}=3.152, p=0.012)$, inner quadrant tumor $(\mathrm{HR}=2.064, \mathrm{p}=0.012)$ and non-luminal subtype $(\mathrm{HR}=$
2.282, $p=0.005$ ) were independent risk factors for LRFS.

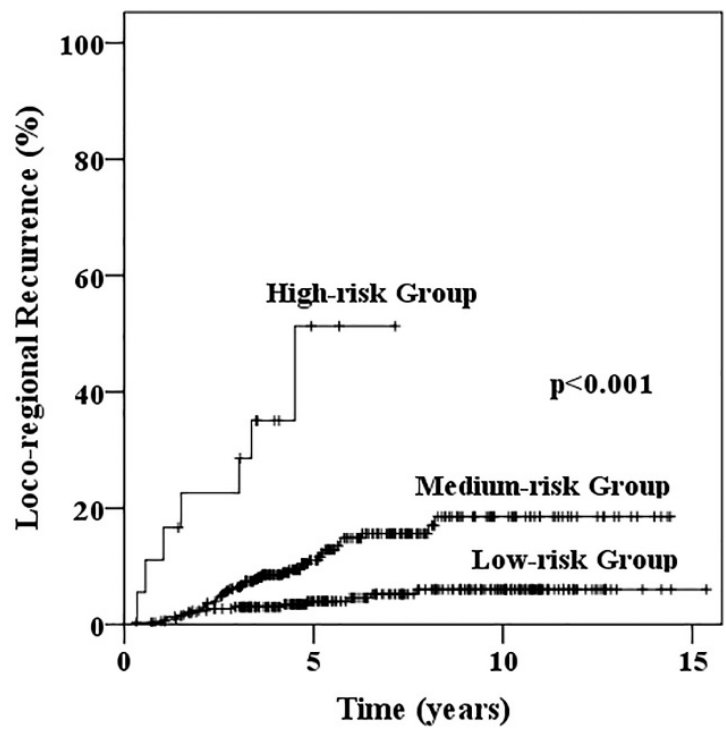

Figure 2. Loco-regional recurrence probability for patients with one to three positive axillary lymphadenopathy stratified into low-, medium- and high-risk loco-regional recurrence.

Table 2. Univariate analysis of prognostic factors with patient characteristics

\begin{tabular}{|c|c|c|c|c|c|c|c|}
\hline Factors & No. & $5 y-\operatorname{LRR}(\%)$ & $\chi^{2}$ & $p$ value & $5 y-L R F S(\%)$ & $\chi^{2}$ & $p$ value \\
\hline \multicolumn{8}{|l|}{ Age, yrs } \\
\hline$\leq 35$ & 70 & 24.2 & 20.070 & $<0.001$ & 74.0 & 10.554 & 0.001 \\
\hline$>35$ & 586 & 6.8 & & & 87.6 & & \\
\hline \multicolumn{8}{|c|}{ Primary tumor site } \\
\hline IQ & 156 & 17.3 & 17.195 & $<0.001$ & 80.0 & 5.225 & 0.073 \\
\hline CQ & 45 & 4.4 & & & 87.8 & & \\
\hline OQ & 455 & 6.0 & & & 88.2 & & \\
\hline \multicolumn{8}{|c|}{ Pathological T Stage } \\
\hline $\mathrm{T}_{1}$ & 247 & 5.6 & 7.424 & 0.006 & 89.9 & 5.182 & 0.023 \\
\hline $\mathrm{T}_{2}$ & 409 & 10.5 & & & 83.9 & & \\
\hline \multicolumn{8}{|c|}{ Number of positive axillary LN } \\
\hline 1 & 367 & 7.1 & 3.867 & 0.049 & 86.9 & 1.544 & 0.214 \\
\hline $2 \sim 3$ & 289 & 10.6 & & & 85.2 & & \\
\hline \multicolumn{8}{|l|}{ p53 status } \\
\hline$<2+$ & 301 & 5.7 & 7.435 & 0.006 & 87.8 & 1.212 & 0.271 \\
\hline$\geq 2+$ & 156 & 13.5 & & & 82.6 & & \\
\hline \multicolumn{8}{|l|}{ Ki 67 status } \\
\hline$<14 \%$ & 108 & 2.4 & 5.691 & 0.017 & 94.7 & 5.421 & 0.020 \\
\hline$\geq 14 \%$ & 308 & 9.6 & & & 84.3 & & \\
\hline \multicolumn{8}{|c|}{ Molecular subtypes } \\
\hline Luminal & 522 & 6.8 & 8.174 & 0.004 & 88.3 & 11.375 & 0.001 \\
\hline Non-Luminal & 134 & 15.9 & & & 78.0 & & \\
\hline
\end{tabular}

\section{Prognosis of patients with different risk factors}

According to the five factors which significant effect on LRR and LRFS (age, primary tumor site, pathological $\mathrm{T}$ stage, Ki67 status and molecular subtypes), all patients were divided into three groups: low-risk group (patients with 0-1 risk factors, $n=302$ ), 
medium-risk group (with 2-3 risk factors, $\mathrm{n}=336$ ) and high-risk group (with $4-5$ risk factors, $n=18$ ). The 5 -year LRR for low-, medium-, high-risk groups were $4.0 \%, 11.1 \%$ and $51.3 \%(\chi 2=48.180, p<0.001$, Fig. 2$)$, 5-year LRFS were $90.5 \%, 84.4 \%$ and $41.7 \%(x 2=42.469$, $p<0.001)$, 5-year distant metastasis free survive (DMFS) were $91.7 \%, 82.5 \%$ and $48.4 \% \quad(x 2=29.937$, $p<0.001)$, and 5 -year OS were $93.0 \%, 89.2 \%$ and $64.2 \%$ $(\chi 2=21.599, \quad p<0.001)$, respectively (Fig. 3).

We further explore the differences of LRR and LRFS in patients with primary tumors in outer or central quadrants and patients with tumor in inner quadrant. There was no significant difference in low-risk group (0-1 risk factors) between two groups, but patients with primary tumor in inner quadrant showed higher LRR and poorer LRFS when risk factors is $\geq 2$ than patients with tumors in outer or central quadrants (Table 3 ).

\section{Discussion}

The main finding of this study is that inner quadrant tumor is an independent predictor for LRR and LRFS in early breast cancer patients with one to three positive axillary lymph nodes. In addition to that, with the increase of risk factors ( $\geq 2$ risk factors), the LRR of patients with tumor in inner quadrant rise rapidly, LRFS significantly reduced, while there is no significant change in patients with primary tumors in outer or central quadrants.

It has been proved that early breast cancer patients with one to three positive axillary lymphadenophy could benefit from postoperative radiotherapy. The result of meta-analysis of 8135 women in 22 randomised trials performed by EBCTCG $^{[12]}$ showed that after mastectomy and axillary dissection, radiotherapy reduced both recurrence and breast cancer mortality in patients with one to three positive lymph nodes even when systemic therapy was given. The result of phase III clinical trial performed by Danish Breast Cancer Research Group (DBCG82b\&c) ${ }^{[15]}$ revealed that the 15-year LRR rate of patients with one to three positive nodes reduced from $27 \%$ to $4 \%$ after receiving postoperative radiotherapy $(p<0.001)$, and the 15 -year OS increased from $48 \%$ to $57 \%(p=0.03)$. In Canada NCIC-CTG MA.20 Research[16], 85\% of patients were with one to three positive lymph nodes and the results showed that the regional lymph node irradiation could significantly improve DFS and OS ( $p=0.003$ and $0.07)$. The latest NCCN clinical guidelines[17] also strongly recommended regional nodal radiation therapy for patients with one to three-positive lymph nodes.
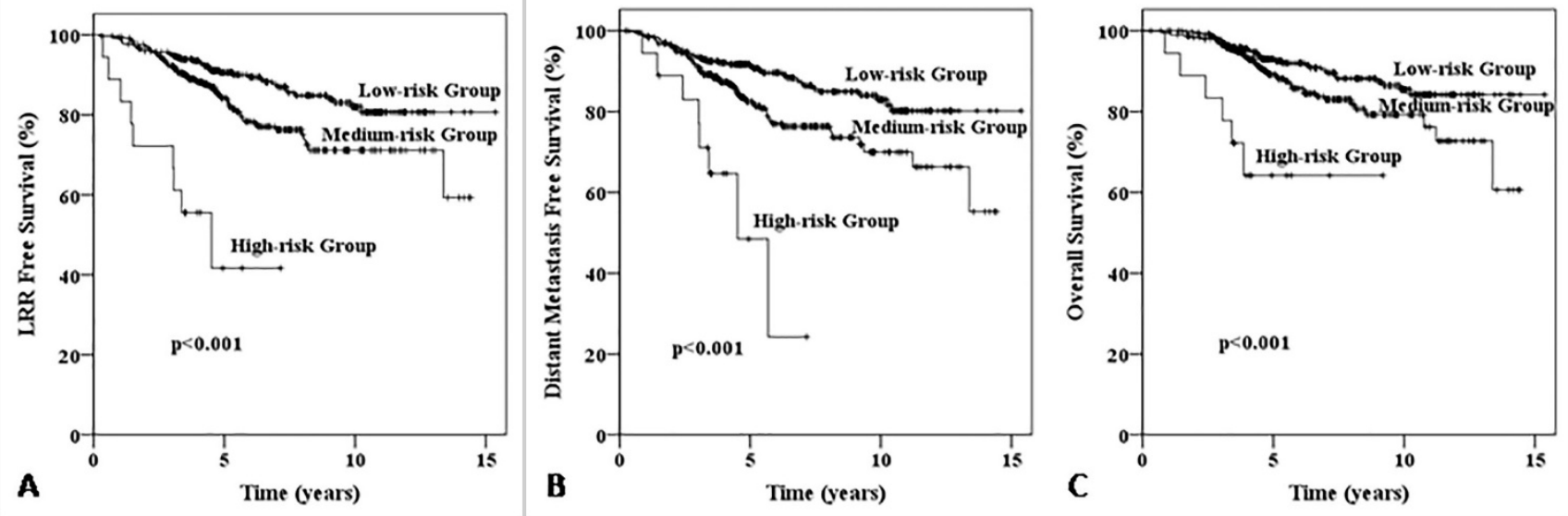

Figure 3. Kaplan-Meier survival curve for patients with one to three positive axillary lymphadenopathy stratified into low-, medium- and high-risk loco-regional recurrence. The LRR free survival (LRFS) curve for patients with one to three positive axillary lymphadenopathy stratified into low-, medium- and high-risk loco-regional recurrence (A); The distant metastasis free survival (DMFS) curve for patients with one to three positive axillary lymphadenopathy stratified into low-, medium-and high-risk loco-regional recurrence (B); The overall survival (OS) curve for patients with one to three positive axillary lymphadenopathy stratified into low-, medium- and high-risk loco-regional recurrence (C).

Table 3. The comparison of LRR and LRFS of 656 patients with different LRR risk classification.

\begin{tabular}{|c|c|c|c|c|c|c|c|c|}
\hline \multirow[t]{2}{*}{ LRR risk classification* } & \multicolumn{2}{|l|}{ NO. } & \multicolumn{3}{|c|}{$5 y-L R R(\%)$} & \multicolumn{3}{|c|}{ 5y-LRFS (\%) } \\
\hline & IQ & CQ/OQ & IQ & CQ/OQ & $p$ value & IQ & CQ/OQ & $p$ value \\
\hline 0 & 21 & 79 & 0 & 2.6 & 0.616 & 100 & 91.9 & 0.270 \\
\hline 1 & 62 & 202 & 10.8 & 5.0 & 0.033 & 89.1 & 89.0 & 0.639 \\
\hline 2 & 58 & 169 & 23.2 & 6.8 & 0.009 & 71.7 & 86.4 & 0.039 \\
\hline$\geq 3$ & 15 & 50 & 56.9 & 11.8 & 0.006 & 35.6 & 84.2 & 0.004 \\
\hline
\end{tabular}

Abbreviations: $\mathrm{IQ}=$ Inner Quadrant, $\mathrm{OQ}=$ Outer Quadrant, $\mathrm{CQ}=$ Central Quadrant

* Primary tumor location was not included 
Previous studies suggested that the high-risk patients should been screened out according to the number of risk factors and receive postoperative radiotherapy because of proportional larger gains. However, there were no consistent results on the predictive value of primary tumor site for loco-recurrence in early breast cancer patients[18-24]. Janni et al. ${ }^{[19]}$ reported that primary tumor site had no significant effect on disease-free survival (DFS) and OS. Jayasinghe et al.[20] reported that the 10-year OS of patients with primary tumor in central quadrant was significantly lower than that of patients with inner and outer quadrant primary tumor, and there was no difference in latter two groups. Gaffney et al. [21] reported that patients with outer tumor location demonstrated superior breast cancer-specific survival (BCSS) and OS compared with patients with inner quadrant location on Kaplan-Meier analysis $(p<0.001)$. Hazrah et al. ${ }^{[22]}$ reported that patients with inner and periareolar quadrant tumor had a higher hazard for systemic disease relapse $(p=0.02$ and $p=0.04$, respectively). Colleoni et al.[23] reported that the 10-year DFS and OS of patients with medial tumors was significant superior to those with non-medial tumors $(p=0.01$ for DFS and $p=0.04$ for OS).

The results of this study showed that inner quadrant tumor is also an independent predictor for LRR and LRFS in early breast cancer patients with one to three positive axillary lymph nodes. The supraclavicular and IMLN LRR rate of patients with primary tumor in inner quadrant was significant higher than that of patients with outer or central quadrant tumor location $(11.4 \%$ vs. $3.7 \%, p<0.001)$, and this may be attributed to the fact that the main lymphatic drainage of inner and central breast is to IMLN and then drainages to the supraclavicular lymph nodes. In clinical work, the monitoring of IMLN often be ignored before or after treatment, which leads to the metastasis of IMLN could not be detected and treated timely. The IMLN detection were not conventionally performed on the patients enrolled in this research, so the conclusion of this study may not apply to the patients who have received IMLN examination before treatment. Another valuable finding in this study is that with the increase of risk factors ( $\geq 2$ risk factors), the LRR of patients with tumor in inner quadrant rise rapidly, LRFS significantly reduced, while there is no significant change in patients with primary tumors in outer or central quadrants, so we recommend that adjuvant radiotherapy should be considered for the former patients in order to reduce the risk of LRR.

The result that the number of detected axillary lymph node had no significant influence on 5-year LRR ( $<10$ vs. $\geq 10)$ s different from previous studies. The reasons are as follows: Firstly, there are certain individual differences in the number of axillary lymph nodes; Secondly, as a single center study, although the number of detected axillary lymph nodes may be different in different periods, the surgeon would perform lymph node dissection according to similar principles, so there was little difference in the actual number of detected lymph nodes.

There are several potential limitations in our study. The main limitation of our study was its retrospective feature, and several different chemotherapy regimens were adopted, which may influence prognosis in a certain extent. Besides, pathologic serial section cutting technique were not adopted in majority patients of this study, which lead to lower detection rates of vessel invasion.

In conclusion, inner quadrant tumor is an independent predictor for LRR and LRFS in patients with early breast cancer and one to three positive axillary lymph nodes, which is more accurately in combination with other prognostic indexes including patient age, pathological $\mathrm{T}$ stage, Ki67 status, molecular subtypes.

\section{Abbreviations}

LRR: loco-regional recurrence; LRFS: LRR-free survival; EBCTCG: The Early Breast Cancer Trialists' Collaborative Group; OQ: outer quadrant; IQ: inner quadrant; CQ: central quadrant; ER/PR: estrogen/ progestogen receptors; FISH: fluorescence in situ hybridization; HER2: human epidermal growth factor receptor 2; TN: triple-negative; CAF: cyclophosphamide and epirubicin/doxorubicin and fluorouracil; TA: docetaxel/taxanes and epirubicin/doxorubicin; CMF: cyclophosphamide, methotrexate and fluorouracil; CA: cyclophosphamide and epirubicin/ doxorubicin; CMO: cyclophosphamide, mitomycin and vinblastine; DM: distant metastasis; IMLN: internal mammary lymphoma nodes; DMFS: distant metastasis free survive; DFS: disease-free survival; BCSS: breast cancer-specific survival.

\section{Acknowledgments}

We thank the medical and radiation oncologists and the pathologists at Sun Yat-sen University Cancer Center for their cooperation and support.

\section{Grant support}

Science and Technology Planning Project of Guangdong Province, China (Grant No. 2012B031800117). 
National Natural Science Foundation of China (Grant No. 81172209).

Natural Science Foundation of Guangdong Province, China (S2011020003612).

\section{Competing Interests}

The authors have declared that no competing interests exist.

\section{References}

1. Nordenskjold AE, Fohlin H, Albertsson P, Arnesson LG, Chamalidou C, Einbeigi Z, et al. No clear effect of postoperative radiotherapy on survival of breast cancer patients with one to three positive nodes: a population-based study. Ann Oncol. 2015; 26: 1149-54.

2. Mazeron JJ. [Should we deliver radiotherapy after mastectomy and axillary surgery in the women with one to three positive lymph nodes?]. Bulletin du cancer. $2015 ; 102: 4$.

3. Huo D, Hou N, Jaskowiak N, Winchester DJ, Winchester DP, Yao K. Use of Postmastectomy Radiotherapy and Survival Rates for Breast Cancer Patients with T1-T2 and One to Three Positive Lymph Nodes. Ann Surg Oncol. 2015.

4. He ZY, Wu SG, Zhou J, Li FY, Lin Q, Lin HX, et al. Postmastectomy radiotherapy improves disease-free survival of high risk of locoregional recurrence breast cancer patients with t1-2 and 1 to 3 positive nodes. PloS one. 2015; 10: e0119105.

5. Sedlmayer F, Sautter-Bihl ML, Budach W, Dunst J, Fastner G, Feyer P, et al. DEGRO practical guidelines: radiotherapy of breast cancer I: radiotherapy following breast conserving therapy for invasive breast cancer. Strahlenther Onkol. 2013; 189: 825-33

6. Katz A, Strom EA, Buchholz TA, Thames HD, Smith CD, Jhingran A, et al. Locoregional recurrence patterns after mastectomy and doxorubicin-based chemotherapy: implications for postoperative irradiation. J Clin Oncol. 2000; 18: $2817-27$.

7. Ragaz J, Olivotto IA, Spinelli JJ, Phillips N, Jackson SM, Wilson KS, et al. Locoregional radiation therapy in patients with high-risk breast cancer receiving adjuvant chemotherapy: 20-year results of the British Columbia randomized trial. Journal of the National Cancer Institute. 2005; 97: 116-26.

8. Woodward WA, Strom EA, Tucker SL, Katz A, McNeese MD, Perkins GH, et al. Locoregional recurrence after doxorubicin-based chemotherapy and postmastectomy: Implications for breast cancer patients with early-stage disease and predictors for recurrence after postmastectomy radiation. Int J Radiat Oncol Biol Phys. 2003; 57: 336-44.

9. Yang PS, Chen CM, Liu MC, Jian JM, Horng CF, Liu MJ, et al. Radiotherapy can decrease locoregional recurrence and increase survival in mastectomy patients with T1 to T2 breast cancer and one to three positive nodes with negative estrogen receptor and positive lymphovascular invasion status. Int J Radiat Oncol Biol Phys. 2010; 77: 516-22.

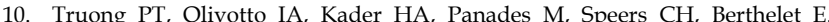
Selecting breast cancer patients with T1-T2 tumors and one to three positive axillary nodes at high postmastectomy locoregional recurrence risk for adjuvant radiotherapy. Int J Radiat Oncol Biol Phys. 2005; 61: 1337-47.

11. Early Breast Cancer Trialists' Collaborative Group. Radiotherapy for early breast cancer. Cochrane Database Syst Rev. 2002: CD003647.

12. Ebctcg, McGale P, Taylor C, Correa C, Cutter D, Duane F, et al. Effect of radiotherapy after mastectomy and axillary surgery on 10 -year recurrence and 20-year breast cancer mortality: meta-analysis of individual patient data for 8135 women in 22 randomised trials. Lancet. 2014; 383: 2127-35.

13. Li FY, Wu SG, Zhou J, Sun JY, Lin Q, Lin HX, et al. Prognostic value of Ki-67 in breast cancer patients with positive axillary lymph nodes: a retrospective cohort study. PLoS One. 2014; 9: e87264.

14. Wiechmann L, Sampson M, Stempel M, Jacks LM, Patil SM, King T, et al. Presenting features of breast cancer differ by molecular subtype. Ann Surg Oncol. 2009; 16: 2705-10

15. Overgaard M, Nielsen HM, Overgaard J. Is the benefit of postmastectomy irradiation limited to patients with four or more positive nodes, as recommended in international consensus reports? A subgroup analysis of the DBCG 82 b\&c randomized trials. Radiother Oncol. 2007; 82: 247-53.

16. Olivotto IA, Chua B, Elliott EA, Parda DS, Pierce LJ, Shepherd L, et al. A clinical trial of breast radiation therapy versus breast plus regional radiation therapy in early-stage breast cancer: the MA20 trial. Clinical breast cancer. $2003 ; 4: 361-3$

17. Gradishar WJ, Anderson BO, Blair SL, Burstein HJ, Cyr A, Elias AD, et al. Breast cancer version 3.2014. Journal of the National Comprehensive Cancer Network : JNCCN. 2014; 12: 542-90.

18. Xue C, Peng RJ, Wang SS, Shi YX, An X, Xu F, et al. Operable breast cancer of the inner hemisphere is associated with poor survival. J Breast Cancer. 2015; 18: 36-43.

19. Janni W, Rack B, Sommer H, Schmidt M, Strobl B, Rjosk D, et al. Intra-mammary tumor location does not influence prognosis but influences the prevalence of axillary lymph-node metastases. J Cancer Res Clin Oncol. 2003; 129: 503-10

20. Jayasinghe UW, Boyages J. Tumour location is not an independent prognostic factor for survival following a diagnosis of breast cancer. Breast. 2009; 18: 41-6.

21. Gaffney DK, Tsodikov A, Wiggins CL. Diminished survival in patients with inner versus outer quadrant breast cancers. J Clin Oncol. 2003; 21: 467-72.

22. Hazrah P, Dhir M, Gupta SD, Deo V, Parshad R. Prognostic significance of location of the primary tumor in operable breast cancers. Indian J Cancer. 2009; 46: 139-45.

23. Colleoni M, Zahrieh D, Gelber RD, Holmberg SB, Mattsson JE, Rudenstam $\mathrm{CM}$, et al. Site of primary tumor has a prognostic role in operable breast cancer: the international breast cancer study group experience. J Clin Oncol. 2005; 23: 1390-400.

24. Wu S, Zhou J, Ren Y, Sun J, Li F, Lin Q, et al. Tumor location is a prognostic factor for survival of Chinese women with T1-2N0M0 breast cancer. Int J Surg. 2014; 12: 394-8. 